\title{
Remote targeted electrical stimulation
}

\author{
Rahul Cheeniyil $^{1}$ and Jan Kubanek ${ }^{1 *}$ \\ ${ }^{1}$ University of Utah, 36 S Wasatch Dr, Salt Lake City, UT 84112 \\ *Corresponding author: jan.kubanek@utah.edu
}

The ability to generate electric fields in deep tissues remotely, without surgically implanting electrodes, could transform diagnoses and treatments of nervous system disorders. Here, we show that focal electrostimulation effects can be elicited remotely by combining two noninvasive forms of energiesmagnetic and focused ultrasonic fields. The approach, based in the Lorentz equation and referred to as Lstim, electrically stimulates specified tissue targets with the precision of deep brain or spinal cord stimulation, but does not require electrode implantation. Lstim potentiated the responses of human nerves, enhancing the neuromodulatory effects of ultrasound by $74 \%$ on average. The effects showed a double dissociation-a significant and substantial increase in nociceptive responses, yet a significant reduction in tactile responses. In line with the Lorentz equation, Lstim was only observed when nerves were oriented perpendicularly to the magnetic and ultrasonic fields. A sham condition showed no effects. Both the ultrasonic and the induced electric fields were well below the respective safety indices, and no detrimental effects were detected. Lstim uniquely integrates noninvasiveness, sharp focus, and the efficacy of electrical stimulation. The approach has the potential to provide a noninvasive tool to dissect brain function in humans and to diagnose the neural circuits involved in nervous system disorders. Moreover, this effect should be taken into account when ultrasound is applied inside MRI.

\section{Introduction}

Nearly one in four people lives with a significant neurological or psychiatric disorder (Lancet, 2017; Ahrnsbrak et al., 2017). Approximately one in three patients across neurological and psychiatric conditions does not respond to drugs or has intolerable side effects (Bystritsky, 2006; Al-Harbi, 2012; Jaffe et al., 2019; Lyons and Pahwa, 2004; Zesiewicz et al., 2010; Elias et al., 2013; Elias et al., 2016; Ferguson, 2001; Karceski, 2007; Louis et al., 2010). Bioelectronic medicine, also referred to as neuromodulation, provides these patients with new treatment options, promising to treat nervous system disorders at their neural source (Perlmutter and Mink, 2006; Arle and Shils, 2017).

Surgery-based electrostimulation approaches, including deep brain stimulation, spinal cord stimulation, sacral nerve stimulation, and vagal nerve stimulation have provided relief to many patients with specific conditions, including movement disorders (Larson, 2014), chronic pain (Lempka and Patil, 2018), bladder control (van Balken et al., 2004), and epilepsy (Elliott et al., 2011), respectively. However, these surgical approaches are costly and incur risks and side effects (Tonge et al., 2015; Bergey et al., 2015; Shah et al., 2015; Sinai et al., 2019; Giordano et al., 2020). Consequently, these approaches are generally limited to an established site of implantation.

Noninvasive approaches, which have rested on electrical, magnetic, electromagnetic, and ultrasonic fields, have much greater flexibility in that they do not incur additional risk to subjects when modulating multiple sites. However, these approaches do not currently have the necessary spatial resolution to modulate specific neural circuits at depth. 
Electric fields generated with current noninvasive approaches, including electroconvulsive therapy (ECT) or transcranial direct or alternating current stimulation (tDCS, tACS) are relatively broad (Lisanby, 2007; CAUMO et al., 2012; Herrmann et al., 2013). Consequently, the sizable activation of the brain associated with ECT often results in cognitive side effects (Ingram et al., 2008). The spatial resolution of these methods can be improved using spatially interfering fields (Nemec, 1959; Grossman et al., 2017), but the resulting fields are still broad within respect to the dimensions of neural circuits.

Transcranial magnetic stimulation (TMS) uses pulses of magnetic fields to noninvasively induce electric fields in the brain. TMS can produce appreciable effects in the cortex and ameliorate symptoms of depression (George et al., 2000), but the approach cannot directly and focally modulate deep brain regions.

Electromagnetic waves currently cannot be used to modulate deep brain targets in a focal manner. At high frequencies (light or infrared), electromagnetic waves are severely attenuated by the skull or superficial tissue layers (McCormick et al., 1992). At lower frequencies, the waves (microwaves) can penetrate into depth, but microwaves at the relevant neuromodulatory doses damage mitochondria and possibly other cellular structures (McRee and Wachtel, 1980; Hao et al., 2015). At yet lower frequencies (radio range), the wavelength is too broad-dozens of centimeters or meters - to allow for focal stimulation (Lustenberger et al., 2013).

Low-intensity focused ultrasound combines depth penetration and safe application. Ultrasound can effectively modulate excitable cells at high frequencies-above $10 \mathrm{MHz}$ - at which there are strong radiation forces that mechanically displace membranes and activate ion channels (Menz et al., 2013; Kubanek et al., 2016; Kubanek et al., 2018; Prieto et al., 2018). However, ultrasound at such high frequencies is severely attenuated by the human skull (Fry, 1977; Fry and Barger, 1978); for this reason, frequencies below $1 \mathrm{MHz}$ have been used for transcranial therapies (Kubanek, 2018). Ultrasound can modulate excitable structures also at lower frequencies (Naor et al., 2016; Blackmore et al., 2019), but strong and reproducible effects, under safe ultrasound levels, remain elusive.

To fill in the current technological gap, we developed and validated an approach that combines the noninvasiveness and targeting capabilities of low-intensity ultrasound with the well-understood and potent effects of electrical stimulation (Cohen and Newsome, 2004; Perlmutter and Mink, 2006; Penfield and Boldrey, 1937; Fried et al., 1991; Suthana et al., 2012). The approach combines two forms of noninvasive energies-ultrasonic and magnetic fields (Figure 1). The idea is based on the fact that motion of a charged molecule $q$ in magnetic field $\vec{B}$ generates Lorentz force $\vec{F}$ on the molecule: $\vec{F}=q(\vec{v} \times \vec{B})$. The generated electrical field has intensity $\vec{E}=\frac{\vec{F}}{q}=\vec{v} \times \vec{B}$. To achieve localized electric field, the movement $\vec{v}$ should occur only in the target of interest. The critical insight is that this can be

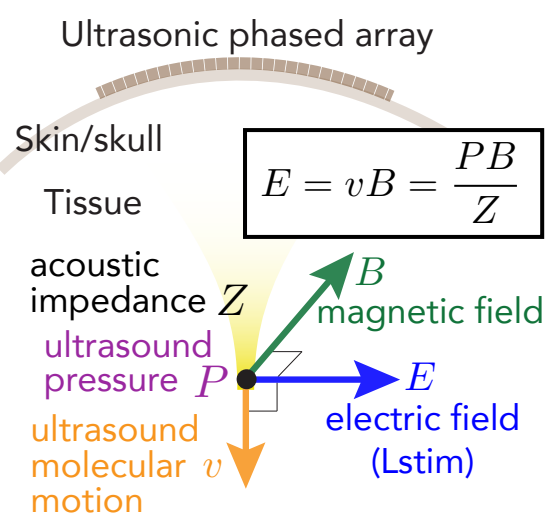

Figure 1. Electrical stimulation without electrodes. An MRI magnet generates magnetic field $B$. An ultrasonic transducer array programmatically focuses ultrasound into a neural circuit of interest. An ultrasound wave, focused into a target with acoustic impedance $Z$, induces in the target motions of molecules with velocity $v=\frac{P}{Z}$. The pressure $P$ (and so the velocity $v$ ) are maximal at the target. When the wave is emitted in a direction perpendicular to $B$, so that the velocity vector is perpendicular to $B$, the target experiences localized electric field $E=\frac{P B}{Z}$. achieved using focused ultrasonic waves. In particular, focused ultrasound induces in its target pressure $P$. This pressure leads to a displacement of molecules at the target, with velocity $\vec{v}$ that points in the direction of the propagating wave. In biological tissues, the speed of the displacement is proportional to the pressure at target, $v=\frac{P}{Z}$, where $Z$ is a tissue constant, "acoustic impedance". Thus, acoustic waves delivered into a target perpendicularly to a magnetic field produce in the target electric field intensity 
$E=\frac{P B}{Z}$. This intensity points in the direction that is perpendicular to both constituents. As a consequence of this electric field, positively and negatively charged molecules are pulled in opposite directions, inducing electric currents. (Ultrasonic fields alone displace positively and negatively charged molecules in the same direction. Therefore, no gradient of charge and so no electric field would be created. The magnetic field is a critical addition.) We refer to this stimulation as "Lstim" given its origin in the Lorentz equation and its electrical and local nature.

A proof of concept of Lstim has been demonstrated in a study that applied weak ultrasound pulses $(\leq 83 \mathrm{kPa})$ to mouse motor cortex in a weak magnetic field $(0.15 \mathrm{~T})$. There were small but significant changes in evoked motor responses of the animals (Wang et al., 2019). Given an effect at a very weak electric field (about $8.3 \mathrm{mV} / \mathrm{m}$ (Wang et al., 2019)), we predict that Lstim using a strong magnetic field will (7 T) will have substantial effects on neural structures.

Mechanistically, the generated electric field $E(t)=\frac{P(t) B(t)}{Z}$ has, for static magnetic field $B$, the same frequency as that of the applied ultrasound, which is generally in the hundreds of kilohertz range. Stimulation at high frequencies rests on a "Gildermeister effect", or integration of membrane potential toward a threshold (Ward, 2009). Moreover, high-frequency electrical stimuli produce a reliable "onset response"-a transient neural response following stimulus onset (Ward, 2009; Grossman et al., 2017). Therefore, when a stimulus is pulsed, we can expect that the onset response will occur within each pulse, thus maximizing the response.

\section{Results}

We applied Lstim to the peripheral nervous system of 18 human subjects (Figure 2). The stimulation was performed inside and outside a $7 \mathrm{~T}$ scanner, with order randomized across the subjects. We tested a total of 9 distinct stimuli-3 distinct amplitudes and 3 distinct waveforms, and included a sham condition (Methods). The ultrasound was delivered into the tissues through a water-filled coupling cone (Figure 2a).

The individual stimuli (Figure 2c) were delivered randomly every 8-12 seconds. The subjects were asked to report any nociceptive or tactile sensation. A nociceptive sensation results from an activation of free nerve endings in the skin (Dubin et al., 2010) and so constitutes a metric of neural activation. The ultrasonic stimuli were safely within the 510 (k) FDA safety indices (FDA, 2019), and the induced electric fields were safely below the recommended charge density (Cogan et al., 2016) of $30 \mu \mathrm{C} / \mathrm{cm}^{2}$ (Figure 3).

We found that ultrasound delivered into the nerves of the subjects' finger inside the magnetic field substantially enhanced the magnitude of nociceptive responses (Figure 4). Across all pressure levels and waveforms, Lstim increased the magnitude of nociceptive responses by $74 \%$. There was a double dissociation of the effects with respect to magnetic field and sensation kind (two-way ANOVA, magnetic field $\times$ sensation interaction, $p<0.001$; $F(1,644)=13.20)$. The effect was similar when the responses were not scaled by their intensity $(p<0.001$; $F(1,644)=13.93)$. Pairwise tests showed that the increase in the nociceptive responses $(p=0.0059 ; t(17)=3.14$, paired two-sided t-test) as well as the decrease in tactile responses ( $p=0.0033 ; t(17)=-3.41)$ were significant. The effects were similar when the responses were not scaled by their intensity $(p=0.0037 ; t(17)=3.36$ and $p=0.0029 ; t(17)=-3.47$, respectively).

We next specifically analyzed the nociceptive responses, which reflect an activation of nerves or nerve endings (Dubin et al., 2010). Figure 5 shows the dependence of all stimuli on the presence or absence of magnetic field, separately for each pressure. The figure confirms the findings of Figure 4 that the magnetic field amplifies the nociceptive responses. We assessed the effects using a full, three-way ANOVA model with factors magnetic field, 


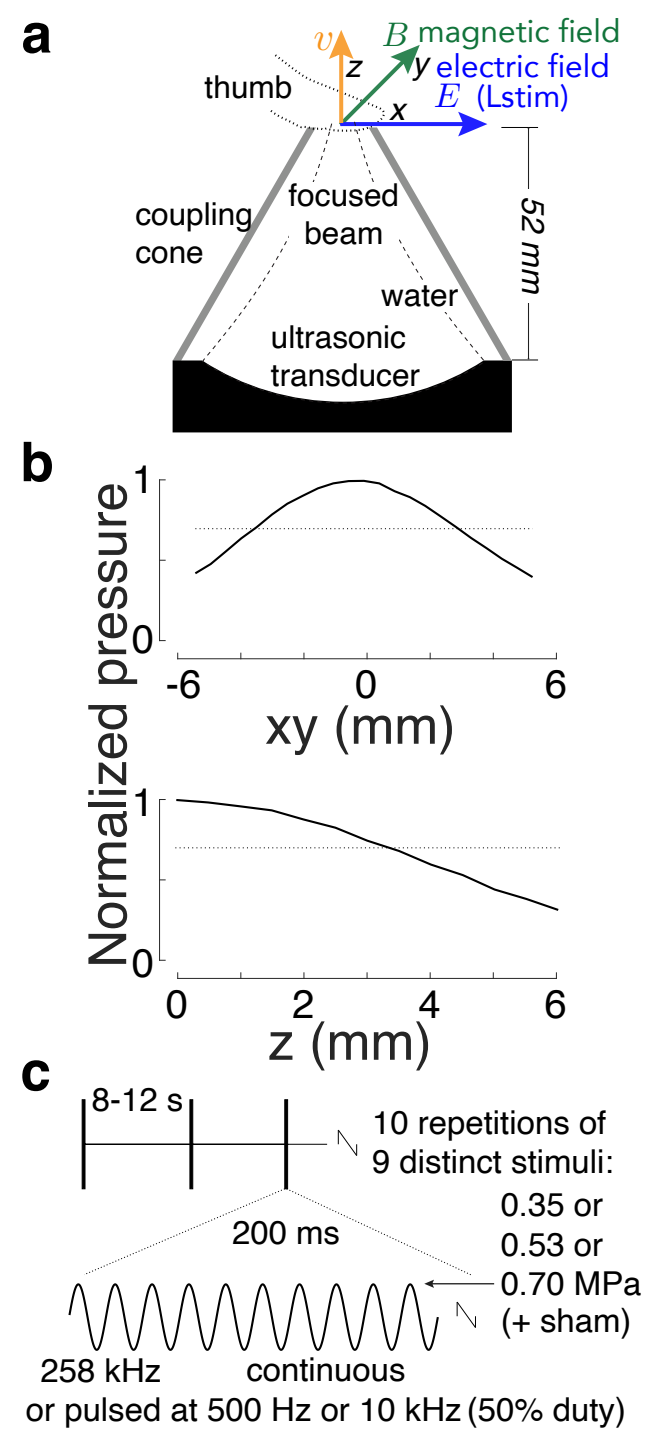

Figure 2. Apparatus and stimuli. a) Apparatus. A focused ultrasound transducer delivered a $258 \mathrm{kHz}$ stimulus into a subject's thumb using a coupling cone filled with degassed water. The stimulation was performed inside a $7 \mathrm{~T}$ scanner or $3 \mathrm{~m}$ away from it. Subjects were instructed to place the thumb in the direction perpendicular to the magnetic and ultrasonic fields, to maximize Lstim-based effects. b) Peaknormalized ultrasound pressure field. The pressure profile was averaged over the $x$ and $y$ dimensions. The dotted lines show the $0.707(0.5)$ pressure (intensity) levels to characterize the fields using full-widthat-half-maximum values. The full-width-athalf-maximum (FWHM) diameter was 6.5 $\mathrm{mm}$, and focal length $3.3 \mathrm{~mm}$. c) Stimuli. Each subject experienced 10 repetitions of 10 distinct stimuli, including sham. The stimuli, $200 \mathrm{~ms}$ in duration, were selected randomly and delivered each 8-12 seconds. We tested 3 pressure levels and continuous and pulsed $(500 \mathrm{~Hz}, 10 \mathrm{kHz}$ frequency, $50 \%$ duty) stimuli. 


\begin{tabular}{|c|c|c|c|c|c|c|c|}
\hline $\begin{array}{c}\text { Pressure } \\
\text { (MPa) }\end{array}$ & $\begin{array}{c}E \\
(V / m)\end{array}$ & Waveform & $\begin{array}{l}\text { On } \\
\text { (ms) }\end{array}$ & $\begin{array}{l}\text { Off } \\
\text { (ms) }\end{array}$ & $\begin{array}{c}I_{S P P A} \\
\left(W / \mathrm{cm}^{2}\right)\end{array}$ & $\begin{array}{c}I_{\text {SPTA }} \\
\left(\mathrm{W} / \mathrm{cm}^{2}\right)\end{array}$ & $\begin{array}{r}\text { Charge } \\
\text { density } \\
\left(\mu \mathrm{C} / \mathrm{cm}^{2}\right)\end{array}$ \\
\hline 0.35 & 1.53 & Pulsed & 100 & 9900 & 3.8 & 0.02 & 1.41 \\
\hline 0.53 & 2.30 & Pulsed & 100 & 9900 & 8.6 & 0.04 & 2.11 \\
\hline 0.70 & 3.06 & Pulsed & 100 & 9900 & 15.3 & 0.08 & 2.82 \\
\hline 0.35 & 1.53 & Continuous & 200 & 9800 & 3.8 & 0.04 & 2.82 \\
\hline 0.53 & 2.30 & Continuous & 200 & 9800 & 8.6 & 0.09 & 4.22 \\
\hline 0.70 & 3.06 & Continuous & 200 & 9800 & 15.3 & 0.15 & 5.63 \\
\hline \multicolumn{5}{|c|}{ Recommended limit } & 190 & 0.72 & 30 \\
\hline
\end{tabular}

Figure 3. Stimuli and their safety. The study used 9 distinct stimuli: 3 levels of pressure, and 3 distinct waveforms, one continuous ( $100 \%$ duty) and two pulsed ( $50 \%$ duty). All stimuli were $200 \mathrm{~ms}$ in duration and were delivered every $10 \mathrm{~s}$ on average. The study followed the FDA 510(k) Track 3 recommendations (FDA, 2019): peak intensity $I_{\mathrm{SPPA}}$ and time-average intensity $I_{\mathrm{SPTA}}$. $E$ is the induced peak Lstim intensity in a $7 \mathrm{~T}$ magnetic field. The computation of the charge density assumes brain conductivity of $0.26 \mathrm{~S} / \mathrm{m}$ (Koessler et al., 2017). Electrical stimulation should ideally not exceed charge density of 30 $\mu \mathrm{C} / \mathrm{cm}^{2}$ (Cogan et al., 2016). Green entries indicate that all stimuli are well within the recommended levels.

ultrasound pressure, stimulus waveform, and all possible interactions (Table 1 ). Indeed, the effect of magnetic field was significant also in this analysis $(p<0.001, F(1,408)=18.55)$.

Lstim produces focused electric fields at ultrasound targets according to $E=\frac{P B}{Z}$. In this equation, the effect increases with the ultrasound pressure $P$. Therefore, the higher the ultrasound pressure, the stronger the induced electric fields, and the stronger the nociceptive responses we should observe, in addition to any neuromodulatory effects of ultrasound alone. In line with this expectation, we found a significant interaction between the magnetic field and ultrasound pressure $(p=0.0012$, $F(3,408)=5.41)$.

We summarize the effects of all factors and interactions in Table 1. With respect to nerve activation, as assessed by the nociceptive responses, there was a significant interaction between magnetic field and the stimulus waveform ( $p=0.043$, $F(2,408)=3.16)$. As expected (see Introduction), the contrast between Lstim and ultrasound only was higher when the ultrasound was pulsed. Specifically, averaged across all pressures, the response frequency ratio ( $7 \mathrm{~T}$ versus $0 \mathrm{~T}$ ) for the continuous waveform was 1.61 , compared to 1.85 and 3.85 for the pulsed $500 \mathrm{~Hz}$ and $10 \mathrm{kHz}$ waveforms, respectively.

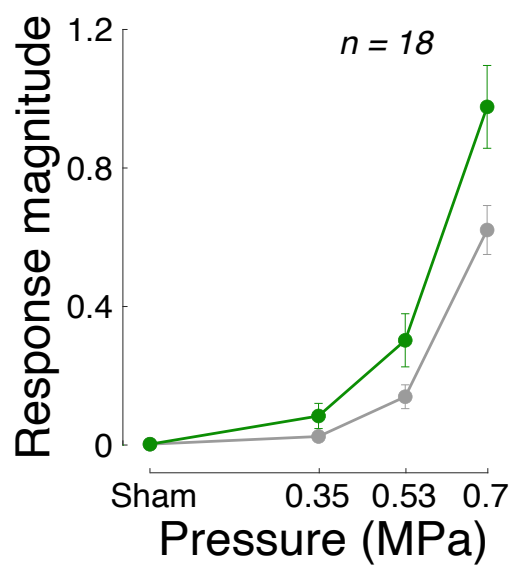

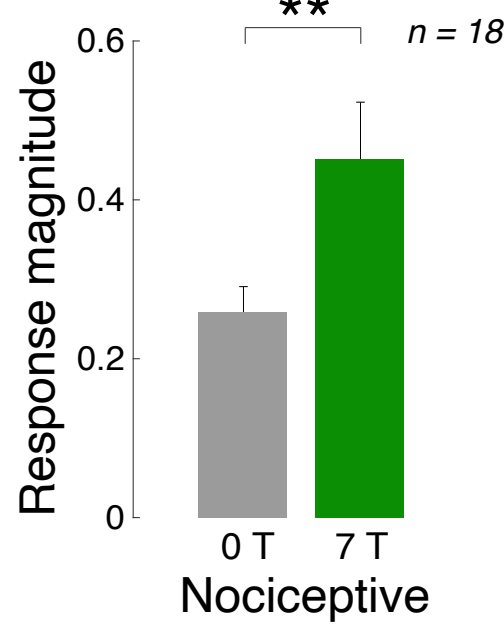

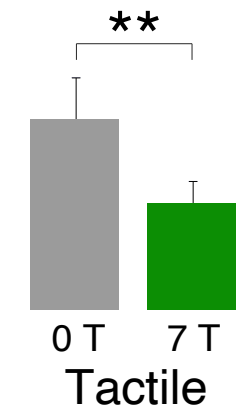

Figure 4. Magnetic field combined with focused ultrasound remotely produces targeted neurostimulatory effects. Mean \pm s.e.m. response magnitude (see Methods) for ultrasound alone (0 T) and ultrasound combined with magnetic field (7 T), separately for nociceptive (left) and tactile (right) responses. Data were pooled over all stimuli tested. The double stars indicate effects significant at $p<0.01$.

Figure 5. The stimulatory effects as a function of ultrasound pressure. Mean \pm s.e.m. magnitude of nociceptive responses as a function of magnetic field and the pressure of ultrasound at target. Data were pooled over all waveforms. 


\begin{tabular}{l|l|l} 
& Nociceptive & Tactile \\
\hline \hline$M$ & $<\mathbf{0 . 0 0 1}$ & $\mathbf{0 . 0 0 4 2}$ \\
$P$ & $<\mathbf{0 . 0 0 1}$ & $<\mathbf{0 . 0 0 1}$ \\
$W$ & $<\mathbf{0 . 0 0 1}$ & $<\mathbf{0 . 0 0 1}$ \\
\hline$M \times P$ & $\mathbf{0 . 0 0 1 2}$ & 0.23 \\
$M \times W$ & $\mathbf{0 . 0 4 3}$ & $\mathbf{0 . 0 4 0}$ \\
$P \times W$ & $<\mathbf{0 . 0 0 1}$ & $<\mathbf{0 . 0 0 1}$ \\
$M \times P \times W$ & 0.54 & 0.83 \\
\hline
\end{tabular}

Table 1. Summary of the effects. The effects of magnetic field $(M)$, ultrasound pressure $(P)$, and stimulus waveform ( $W$; continuous or pulsed) on the frequency of nociceptive (left column) and tactile responses (right column). These effects were assessed using a threeway ANOVA that featured the three main effects and all possible interactions. Bold entries are significant $(p<0.05)$.

If the reported effects are indeed due to the induction of localized electric field, as governed by the Lorentz electromotive force equation, they should strongly depend on the orientation of the finger with respect to the electric field. Specifically, electric fields can effectively stimulate nerves if their gradients point along nerves, as opposed to across (Rattay, 1999). To test this, 4 subjects were asked to place their thumb on the aperture 1) perpendicularly to the magnetic field (up until now) 2) in parallel with the magnetic field. We found (Figure 6) that these conditions significantly modulated the responses $(p=0.041, F(2,33)=3.5)$. However, as expected, the effect was specific to the perpendicular geometry; there was no effect for the parallel geometry $(p=0.88$, $t(3)=0.17$, paired two-sided t-test).

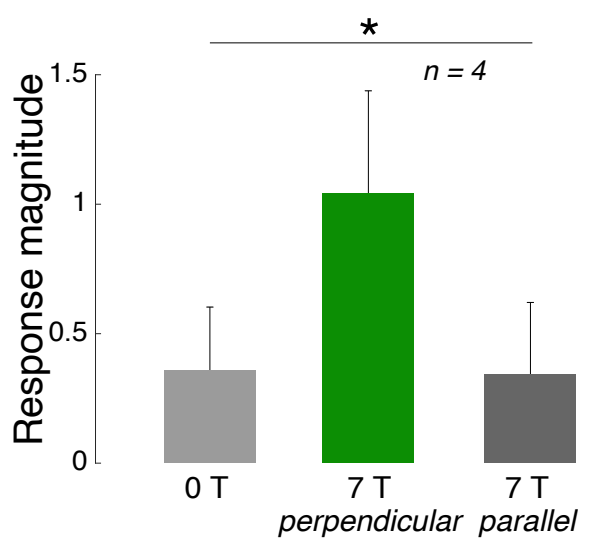

Figure 6. Lstim stimulates nerves in an orientation-specific manner. Mean \pm s.e.m. magnitude of nociceptive responses as a function of the orientation of the induced electric field with respect to the subjects' nerves. The neuromodulatory effects are maximized when nerves in the subjects' thumb are aligned with the induced electric field (green). Data were pooled over all waveforms tested. Star: the modulation by the magnetic field and its orientation was significant $(p<0.05)$.

\section{Discussion}

In this study, we applied and tested an approach that induces focal electric fields in remote targets. The approach, Lstim, rests on the Lorentz effect-movement of charged particles in strong magnetic field. Critically, in the Lstim embodiment of the Lorentz effect, the movement is induced by cycle-by-cycle displacement of particles at the target of focused ultrasound. We found that the combination of ultrasonic and magnetic fields safely produced effects that were about 1.7 times stronger compared to those induced by ultrasound alone. As expected by the governing equation $\left(E=\frac{P B}{Z}\right)$, we found that the effects scaled as a function of the ultrasound pressure. In addition, the effects followed the direction-specific Lorentz equation. No effects were observed for the sham condition. Together, these findings confirm the hypothesis that a remote generation of focal electric fields by combining ultrasonic and magnetic fields is indeed feasible.

The produced electric field is deterministic, governed solely by the analytic expression $E=\frac{P B}{Z}$ for cases in which the ultrasonic and magnetic fields are perpendicular. (Otherwise, the equation is multiplied by the 
sine of the angle between the two fields.) The acoustic pressure and its waveform $P(t)$ can be measured for a given transducer hardware using a hydrophone. The static magnetic field of MRI scanners is near-homogeneous within an organ (e.g., brain), and so $B$ is also a deterministic quantity (e.g., $7 \mathrm{~T}$ ). The acoustic impedance tissue constant $Z$ is close to water and varies only within about $10 \%$ across soft tissues (Cobbold, 2006; Azhari, 2010). For the brain, the acoustic impedance is about 1.6 MRayl (Azhari, 2010). Using this equation, we can therefore accurately compute the generated electric field intensity. For example, a $0.7 \mathrm{MPa}$ stimulus at a target inside a 7 T MRI scanner, used here, evoked peak electric intensity of about $3.1 \mathrm{~V} / \mathrm{m}$. A field of $3 \mathrm{~V} / \mathrm{m}$ is deemed to be high enough to appreciably influence neural activity (Francis et al., 2003; Liu et al., 2018). Fields as low $0.3 \mathrm{~V} / \mathrm{m}$ have been shown to modulate neuronal spiking (Francis et al., 2003). For instance, transcranial electrical stimulation, for the generally accepted maximum current of $2 \mathrm{~mA}$, produces about $0.28 \mathrm{~V} / \mathrm{m}(95 \mathrm{th}$ percentile) in the brain (Huang et al., 2017). With Lstim, we elicited up to 11 times that intensity while complying with the applicable safety indices (Figure 3).

The effects on nerves were observed already for a relative low ultrasound pressure ( $0.7 \mathrm{MPa}$ in amplitude at focus). We limited the highest pressure level to 0.7 MPa to avoid strong nociceptive sensations. No subject lifted their finger from the aperture during the experiment, and no harmful effects were detected by the experimenter or reported by the subjects. In regard to safety, the FDA $510(\mathrm{k}) I_{\mathrm{SPPA}}$ ultrasound index would allow us to apply up to 2.4 MPa ( $<190 \mathrm{~W} / \mathrm{cm}^{2}$ in soft tissues). At these levels, still considered safe, the effects of Lstim could be expected to be more than 3 times stronger than those reported here. Moreover, for the relatively low frequencies such as those used here, it is conceivable that ultrasound amplitudes higher than 2.4 MPa could be applied in brief pulses without a risk of heating or cavitation (Nightingale et al., 2015). If even stronger effects are needed for certain applications, the stimulation could be performed in stronger magnetic fields $B$. For example, in a $11 \mathrm{~T}$ scanner, a 2.4 MPa ultrasound stimulus would produce a peak intensity of about $16.5 \mathrm{~V} / \mathrm{m}$ at focus, more than 5 times higher than that produced here. In addition, neurons are generally more sensitive to electrical stimuli than nerves. Therefore, Lstim is expected to elicit particularly strong effects when applied to neural circuits.

In fact, Lstim may have been adventitiously at play in the brain already. We revisited the ultrasonic neuromodulation literature (Naor et al., 2016; Kubanek, 2018; Blackmore et al., 2019) with a special focus on the presence of magnetic field. We now make the observation that when subjects are inside MRI, ultrasound can elicit strong effects including phosphenes (Lee et al., 2016) and can have appreciable effects on neural activity in humans ( $\mathrm{Ai}$ et al., 2018). The unbeknown presence of Lstim may thus explain the notable effects in some "ultrasonic neuromodulation" studies.

In addition, several recent studies applied ultrasound of comparable frequency and pressure level to specific regions of the monkey brain inside a $3 \mathrm{~T}$ magnetic field. It has been found that only 40 seconds of such stimulation could dissociate network connectivity in deep brain regions including the amygdala, and these neuroplastic effects outlive the stimulation for about two hours (Verhagen et al., 2019; Fouragnan et al., 2019; Khalighinejad et al., 2020). In many of the cases, the ultrasound was applied to the brain near-perpendicularly to the magnetic field. Given the findings of the present study, therefore, these studies have, at least in part, invoked Lstim. To what extent Lstim contributed to the effects should be investigated in future studies.

There is additional evidence that the effects of Lstim can be durable and so potentially also useful for treatments. Specifically, when subjects move their head near an MRI scanner (1 T field) for $16 \mathrm{~s}$, there can be substantial effects on cognition (van Nierop et al., 2012). This is even though the head motion was about 10 times slower than the molecular motion induced with ultrasound for our strongest stimulus (Figure 3 ). The peak velocity of the head movements in that study is estimated to be $v=0.35 \mathrm{~m} / \mathrm{s}$, assuming average head circumference of 0.56 
m. In $B=1 \mathrm{~T}$, this yields up to $E=0.35 \mathrm{~V} / \mathrm{m}$. These effects were noted about $90 \mathrm{~s}$ following the head motion, indicating that the Lorentz-based effects can outlive their initiation.

Lstim uniquely combines noninvasiveness, sharp focus, targeting flexibility, and the efficacy of electrical stimulation. No other existing approach has these features. This intersection of desirable features is a result of favorable properties of the two constituting fields. Both magnetic and ultrasonic fields are non-invasive, and the hardware for both is readily available in the form of ultrasonic transducers and MRI scanners, respectively. Lstim owes its sharp focus to focused ultrasound. The spatial resolution of the method is in theory comparable to the voxel size of standard imaging transducer systems. For brain applications, existing hardware is able to focus ultrasound, through the human skull, into deep brain circuits that span only about $3 \mathrm{~mm}$ in diameter (Ghanouni et al., 2015). Furthermore, these existing transducer system target ultrasound flexibly, in software. Since ultrasound reaches a target in microseconds, the theoretical temporal resolution of Lstim thus reaches tens-hundreds of microseconds.

Once optimized in regard to stimulation waveforms, this new mode of noninvasive targeted stimulation may develop into a new tool to manipulate neural circuits in the human brain. Such an approach could provide the sorely needed diagnostic tools. For instance, clinical teams could use Lstim to systematically identify the neural circuits that are most strongly involved in specific signs and symptoms in a given patient. The identified circuits could subsequently be modulated using existing clinical tools such as deep brain stimulation or spinal cord stimulation, now applied in a targeted, personalized manner. Analogously, Lstim could be applied in basic neuroscience research as a causal tool to manipulate neural circuits in the human brain. As noted above, ultrasound has been applied to the human brain inside strong magnetic fields ( $3 \mathrm{~T}$ and $7 \mathrm{~T}$ ) already. These envisioned applications are therefore expected to be safe.

Although we have primarily focused on medical applications, Lstim could be applied to remotely induce electric fields also in other disciplines. For example, the method could be used to remotely catalyze chemical reactions in situations in which a sample cannot be influenced by the insertion of metallic electrodes. Food processing, and remote stimulation of sterile tissue or cell cultures constitute additional examples.

In summary, we asked whether the combination of ultrasonic and magnetic fields could provide a noninvasive alternative to existing deep tissue electrical stimulation methods. This study provides an initial proof of concept using intact human peripheral nervous system. The approach, once optimized for specific kinds of excitable structures, may provide the long-sought tool to manipulate the nervous system in humans noninvasively and in a targeted manner.

\section{Methods}

\section{Subjects and apparatus}

The study was approved by the Institutional Review Board of the University of Utah. Eighteen subjects (6 females, 12 males, aged between 21-38 years) participated in the study. All subjects provided an informed consent.

Subjects were asked to gently rest the thumb of their right hand on a plastic coupling cone filled with degassed water (Figure 2a). The height of the cone was $52 \mathrm{~mm}$ and its total diameter $70 \mathrm{~mm}$. The aperture $(1 \mathrm{~mm}$-thick plastic) had a $16 \mathrm{~mm}$ in diameter opening for the ultrasound beam. A focused, MRI-compatible ultrasonic transducer (H-115, Sonic Concepts, $64 \mathrm{~mm}$ diameter, $52 \mathrm{~mm}$ focal depth) was positioned $52 \mathrm{~mm}$ below the aperture (Figure 2a). The transducer was operated at $258 \mathrm{kHz}$. Stimuli were generated by a custom Matlab 
program that produced the stimulation waveforms in a programmable function generator (33520b, Keysight). The signals were amplified using a 55-dB, $258 \mathrm{kHz}-30 \mathrm{MHz}$ power amplifier (A150, Electronics \& Innovation).

Subjects had their eyes closed and wore noise-cancelling earmuffs ( $\mathrm{X} 4 \mathrm{~A}, 3 \mathrm{M} ; 27 \mathrm{~dB}$ noise reduction) so that they could fully focus on the stimuli. Subjects could not hear or see the stimuli or their generation.

\section{Stimuli}

The stimulation was performed inside the bore of a $7 \mathrm{~T} \mathrm{MRI} \mathrm{scanner} \mathrm{(Bruker} \mathrm{BioSpec)} \mathrm{or} \mathrm{at} \mathrm{a} 3 \mathrm{~m}$ distance away from it. The stimulation order was randomized, without replacement, such that half of the subjects experienced the stimulation in the scanner first and the other half near the scanner first. Subjects were asked to place the finger on the aperture in the direction perpendicular to the ultrasonic and magnetic fields (Figure 2a) to maximize the Lstim effects.

We used 9 distinct stimuli, of 3 pressure levels and 3 distinct waveforms. A tenth, sham stimulus, delivered negligible pressure ( $5 \mathrm{kPa}$, corresponding to the noise level of the amplifier-transducer output) under the same conditions. The parameters were chosen to provide safe and effective stimulation. The relatively low carrier frequency $(258 \mathrm{kHz})$ was chosen to maximize the Gildermeister effect (integration of membrane potential toward a threshold), which is more effective at lower frequencies (Ward, 2009). The duration of each stimulus (200 ms) was chosen to provide ample time for the integration. The peak pressure amplitudes of the ultrasound measured at the center of the aperture were $0.35 \mathrm{MPa}, 0.53 \mathrm{MPa}$, and $0.7 \mathrm{MPa}$. The peak pressures were chosen such as to trigger appreciable electric intensities at target (up to $3.1 \mathrm{~V} / \mathrm{m}$ ), but low enough to comply with the $I_{\mathrm{SPPA}}$ Track $3510(\mathrm{k})$ recommendation for each pulse and within the $I_{\text {SPTA }}$ recommendation over the course of the experiment (see Stimulus safety), and low enough to prevent unpleasant nociceptive responses. The stimuli were either continuous (200 ms of tone burst) or pulsed at $500 \mathrm{~Hz}$ or $10 \mathrm{kHz}$ both $50 \%$ duty.

The pressure fields were measured using a capsule hydrophone (HGL-0200, Onda) calibrated between $250 \mathrm{kHz}$ and $40 \mathrm{MHz}$ and secured to 3-degree-of-freedom programmable translation system (Aims III, Onda). There were 10 repetitions of the 10 stimuli, producing a total of 100 stimulation trials per subject inside the scanner and 100 trials outside the scanner. The stimuli were delivered every 8-12 s. The stimuli were drawn from the 100-stimulus set randomly without replacement. This way, stimulus order could not affect the results.

\section{Responses and their assessment}

Subjects were instructed to report a percept with a verbal command of any combination of \{Pain, Vibration, Tap\}, and their intensity (1: low, 2: medium, 3: high). Following each stimulus, the experimenter was prompted to entered the reported sensation (or lack thereof) and its intensity into a command line of the same Matlab program that scheduled the stimuli. The experimenter was blinded to the stimuli. Following the experiment, for each stimulus type, the response magnitude was computed as the proportion of trials in which subjects' registered a response, weighted by the reported intensity. Vibration and tap responses were grouped together as tactile.

\section{Acoustic continuum}

The acoustic impedance of water and skin, including soft tissues, are closely matched (1.48 MRayl compared to 1.68 MRayl (Kuhn et al., 2008)). This way, about $99.6 \%$ of the energy, $1-R^{2}=1-\left(\frac{1.68-1.48}{1.68+1.48}\right)^{2}$, was delivered 
into the finger. The water-finger interface is therefore essentially acoustically transparent and can be considered as a continuum from the perspective of ultrasound.

\section{Stimulus safety}

The ultrasonic stimuli used in this study were safely below the FDA 510(k) Track 3 recommendations (FDA, 2019). In particular, the highest peak pressure used in the study, $0.7 \mathrm{MPa}$, corresponds to peak intensity of $15.3 \mathrm{~W} / \mathrm{cm}^{2}$, which is well below the FDA recommendation of $I_{\text {SPPA }}=190 \mathrm{~W} / \mathrm{cm}^{2}$ (Figure 3). In addition, the time-average spatial peak intensity was $I_{\mathrm{SPTA}}=150 \mathrm{~mW} / \mathrm{cm}^{2}$, also below the FDA recommendation of $I_{\mathrm{SPTA}}=720 \mathrm{~mW} / \mathrm{cm}^{2}$. The computation of the charge density (Figure 3) assumed brain conductivity of $0.26 \mathrm{~S} / \mathrm{m}$ (Koessler et al., 2017). Thus, stimuli of much higher levels could be used, from both the ultrasound safety and electrical stimulation safety perspectives. The 0.7 MPa maximum allowed all sensations to be well tolerated by the subjects. No subject lifted their finger from the aperture during the experiment. No harmful effects were detected by the experimenter or reported by the subjects.

Ahrnsbrak R, Bose J, Hedden S, Lipari R, Park-Lee E (2017) Key substance use and mental health indicators in the United States: Results from the 2016 National Survey on Drug Use and Health. Center for Behavioral Health Statistics and Quality, Substance Abuse and Mental Health Services Administration: Rockville, MD, USA.

Ai L, Bansal P, Mueller JK, Legon W (2018) Effects of transcranial focused ultrasound on human primary motor cortex using $7 \mathrm{t}$ fmri: a pilot study. BMC neuroscience 19:1-10.

Al-Harbi KS (2012) Treatment-resistant depression: therapeutic trends, challenges, and future directions. Patient preference and adherence 6:369.

Arle J, Shils JL (2017) Innovative Neuromodulation Academic Press.

Azhari H (2010) Basics of biomedical ultrasound for engineers John Wiley \& Sons.

Bergey GK, Morrell MJ, Mizrahi EM, Goldman A, King-Stephens D, Nair D, Srinivasan S, Jobst B, Gross RE, Shields DC et al. (2015) Long-term treatment with responsive brain stimulation in adults with refractory partial seizures. Neurology 84:810-817.

Blackmore J, Shrivastava S, Sallet J, Butler CR, Cleveland RO (2019) Ultrasound neuromodulation: A review of results, mechanisms and safety. Ultrasound in medicine \& biology 45:1509-1536.

Bystritsky A (2006) Treatment-resistant anxiety disorders. Molecular psychiatry 11:805-814.

CAUMO W, Souza IC, Torres IL, Medeiros L, Souza A, Deitos A, Vidor L, Fregni F, Volz MS (2012) Neurobiological effects of transcranial direct current stimulation: a review. Frontiers in psychiatry 3:110.

Cobbold RS (2006) Foundations of biomedical ultrasound Oxford university press.

Cogan SF, Ludwig KA, Welle CG, Takmakov P (2016) Tissue damage thresholds during therapeutic electrical stimulation. Journal of neural engineering 13:021001.

Cohen MR, Newsome WT (2004) What electrical microstimulation has revealed about the neural basis of cognition. Current opinion in neurobiology 14:169-177. 
Dubin AE, Patapoutian A et al. (2010) Nociceptors: the sensors of the pain pathway. The Journal of clinical investigation 120:3760-3772.

Elias WJ, Huss D, Voss T, Loomba J, Khaled M, Zadicario E, Frysinger RC, Sperling SA, Wylie S, Monteith SJ et al. (2013) A pilot study of focused ultrasound thalamotomy for essential tremor. New England Journal of Medicine 369:640-648.

Elias WJ, Lipsman N, Ondo WG, Ghanouni P, Kim YG, Lee W, Schwartz M, Hynynen K, Lozano AM, Shah BB et al. (2016) A randomized trial of focused ultrasound thalamotomy for essential tremor. New England Journal of Medicine 375:730-739. Elliott RE, Morsi A, Tanweer O, Grobelny B, Geller E, Carlson C, Devinsky O, Doyle WK (2011) Efficacy of vagus nerve stimulation over time: review of 65 consecutive patients with treatment-resistant epilepsy treated with vns $>10$ years. Epilepsy \& Behavior 20:478-483.

FDA (2019) Marketing clearance of diagnostic ultrasound systems and transducers. Food and Drug Administration FDA2017-D-5372.

Ferguson JM (2001) Ssri antidepressant medications: adverse effects and tolerability. Primary care companion to the Journal of clinical psychiatry 3:22.

Fouragnan EF, Chau BK, Folloni D, Kolling N, Verhagen L, Klein-Flügge M, Tankelevitch L, Papageorgiou GK, Aubry JF, Sallet J et al. (2019) The macaque anterior cingulate cortex translates counterfactual choice value into actual behavioral change. Nature neuroscience 22:797-808.

Francis JT, Gluckman BJ, Schiff SJ (2003) Sensitivity of neurons to weak electric fields. Journal of Neuroscience 23:7255-7261.

Fried I, Katz A, McCarthy G, Sass KJ, Williamson P, Spencer SS, Spencer DD (1991) Functional organization of human supplementary motor cortex studied by electrical stimulation. Journal of Neuroscience 11:3656-3666.

Fry F (1977) Transkull transmission of an intense focused ultrasonic beam. Ultrasound in medicine \& biology 3:179-184. Fry FJ, Barger JE (1978) Acoustical properties of the human skull. The Journal of the Acoustical Society of America 63:1576-1590.

George MS, Nahas Z, Molloy M, Speer AM, Oliver NC, Li XB, Arana GW, Risch SC, Ballenger JC (2000) A controlled trial of daily left prefrontal cortex tms for treating depression. Biological psychiatry 48:962-970.

Ghanouni P, Pauly KB, Elias WJ, Henderson J, Sheehan J, Monteith S, Wintermark M (2015) Transcranial MRI-guided focused ultrasound: a review of the technologic and neurologic applications. American Journal of Roentgenology 205:150-159.

Giordano M, Caccavella VM, Zaed I, Manzillo LF, Montano N, Olivi A, Polli FM (2020) Comparison between deep brain stimulation and magnetic resonance-guided focused ultrasound in the treatment of essential tremor: a systematic review and pooled analysis of functional outcomes. Journal of Neurology, Neurosurgery \& Psychiatry 91:1270-1278.

Grossman N, Bono D, Dedic N, Kodandaramaiah SB, Rudenko A, Suk HJ, Cassara AM, Neufeld E, Kuster N, Tsai LH et al. (2017) Noninvasive deep brain stimulation via temporally interfering electric fields. Cell 169:1029-1041.

Hao YH, Zhao L, Peng RY (2015) Effects of microwave radiation on brain energy metabolism and related mechanisms. Military medical research 2:1-8. 
Herrmann CS, Rach S, Neuling T, Strüber D (2013) Transcranial alternating current stimulation: a review of the underlying mechanisms and modulation of cognitive processes. Frontiers in human neuroscience 7:279.

Huang Y, Liu AA, Lafon B, Friedman D, Dayan M, Wang X, Bikson M, Doyle WK, Devinsky O, Parra LC (2017) Measurements and models of electric fields in the in vivo human brain during transcranial electric stimulation. elife 6:e18834.

Ingram A, Saling MM, Schweitzer I (2008) Cognitive side effects of brief pulse electroconvulsive therapy: a review. The journal of ECT 24:3-9.

Jaffe DH, Rive B, Denee TR (2019) The humanistic and economic burden of treatment-resistant depression in europe: a cross-sectional study. BMC psychiatry 19:247.

Karceski SC (2007) Seizure medications and their side effects. Neurology 69:E27-E29.

Khalighinejad N, Bongioanni A, Verhagen L, Folloni D, Attali D, Aubry JF, Sallet J, Rushworth MF (2020) A basal forebrain-cingulate circuit in macaques decides it is time to act. Neuron 105:370-384.

Koessler L, Colnat-Coulbois S, Cecchin T, Hofmanis J, Dmochowski JP, Norcia AM, Maillard LG (2017) In-vivo measurements of human brain tissue conductivity using focal electrical current injection through intracerebral multicontact electrodes. Human brain mapping 38:974-986.

Kubanek J (2018) Neuromodulation with transcranial focused ultrasound. Neurosurgical Focus 44:E14.

Kubanek J, Shi J, Marsh J, Chen D, Deng C, Cui J (2016) Ultrasound modulates ion channel currents. Scientific Reports 6:24170.

Kubanek J, Shukla P, Das A, Baccus SA, Goodman MB (2018) Ultrasound elicits behavioral responses through mechanical effects on neurons and ion channels in a simple nervous system. Journal of Neuroscience pp. 1458-17.

Kuhn C, Angehrn F, Sonnabend O, Voss A (2008) Impact of extracorporeal shock waves on the human skin with cellulite: a case study of an unique instance. Clinical interventions in aging 3:201.

Lancet (2017) Life, death, and disability in 2016. The Lancet 390:1084-1423.

Larson PS (2014) Deep brain stimulation for movement disorders. Neurotherapeutics 11:465-474.

Lee W, Kim HC, Jung Y, Chung YA, Song IU, Lee JH, Yoo SS (2016) Transcranial focused ultrasound stimulation of human primary visual cortex. Scientific Reports 6.

Lempka SF, Patil PG (2018) Innovations in spinal cord stimulation for pain. Current opinion in biomedical engineering 8:51-60. Lisanby SH (2007) Electroconvulsive therapy for depression. New England Journal of Medicine 357:1939-1945.

Liu A, Vöröslakos M, Kronberg G, Henin S, Krause MR, Huang Y, Opitz A, Mehta A, Pack CC, Krekelberg B et al. (2018) Immediate neurophysiological effects of transcranial electrical stimulation. Nature communications 9:1-12.

Louis ED, Rios E, Henchcliffe C (2010) How are we doing with the treatment of essential tremor (et)? persistence of patients with et on medication: data from 528 patients in three settings. European journal of neurology 17:882-884.

Lustenberger C, Murbach M, Dürr R, Schmid MR, Kuster N, Achermann P, Huber R (2013) Stimulation of the brain with radiofrequency electromagnetic field pulses affects sleep-dependent performance improvement. Brain stimulation 6:805-811. 
Lyons KE, Pahwa R (2004) Deep brain stimulation and essential tremor. Journal of clinical neurophysiology 21:2-5.

McCormick PW, Stewart M, Lewis G, Dujovny M, Ausman Jl (1992) Intracerebral penetration of infrared light. Journal of neurosurgery 76:315-318.

McRee DI, Wachtel H (1980) The effects of microwave radiation on the vitality of isolated frog sciatic nerves. Radiation research 82:536-546.

Menz MD, Oralkan Ö, Khuri-Yakub PT, Baccus SA (2013) Precise neural stimulation in the retina using focused ultrasound. The Journal of Neuroscience 33:4550-4560.

Naor O, Krupa S, Shoham S (2016) Ultrasonic neuromodulation. Journal of Neural Engineering 13:031003.

Nemec H (1959) Interferential therapy: a new approach in physical medicine. Br J Physiother 12:9-12.

Nightingale KR, Church CC, Harris G, Wear KA, Bailey MR, Carson PL, Jiang H, Sandstrom KL, Szabo TL, Ziskin MC (2015) Conditionally increased acoustic pressures in nonfetal diagnostic ultrasound examinations without contrast agents: $A$ preliminary assessment. Journal of Ultrasound in Medicine 34:1-41.

Penfield W, Boldrey E (1937) Somatic motor and sensory representation in the cerebral cortex of man as studied by electrical stimulation. Brain 60:389-443.

Perlmutter JS, Mink JW (2006) Deep brain stimulation. Annu. Rev. Neurosci. 29:229-257.

Prieto ML, Firouzi K, Khuri-Yakub BT, Maduke M (2018) Activation of piezo1 but not nav1. 2 channels by ultrasound at $43 \mathrm{mhz}$. Ultrasound in medicine \& biology 44:1217-1232.

Rattay F (1999) The basic mechanism for the electrical stimulation of the nervous system. Neuroscience 89:335-346.

Shah B, Voss T, Huss D, Elias WJ, Harrison M, Loomba J, Khaled M, Frysinger R (2015) Unilateral focused ultrasound thalamotomy in essential tremor: 3 year safety and efficacy data (p5. 279).

Sinai A, Nassar M, Eran A, Constantinescu M, Zaaroor M, Sprecher E, Schlesinger I (2019) Magnetic resonance-guided focused ultrasound thalamotomy for essential tremor: a 5-year single-center experience. Journal of Neurosurgery 1:1-8.

Suthana N, Haneef Z, Stern J, Mukamel R, Behnke E, Knowlton B, Fried I (2012) Memory enhancement and deep-brain stimulation of the entorhinal area. New England Journal of Medicine 366:502-510.

Tonge M, Ackermans L, Kocabicak E, van Kranen-Mastenbroek V, Kuijf M, Oosterloo M, Kubben P, Temel Y (2015) A detailed analysis of intracerebral hemorrhages in dbs surgeries. Clinical neurology and neurosurgery 139:183-187.

van Balken MR, Vergunst H, Bemelmans BL (2004) The use of electrical devices for the treatment of bladder dysfunction: a review of methods. The Journal of urology 172:846-851.

van Nierop LE, Slottje P, van Zandvoort MJ, de Vocht F, Kromhout H (2012) Effects of magnetic stray fields from a 7 tesla mri scanner on neurocognition: a double-blind randomised crossover study. Occupational and environmental medicine 69:759-766.

Verhagen L, Gallea C, Folloni D, Constans C, Jensen DE, Ahnine H, Roumazeilles L, Santin M, Ahmed B, Lehéricy S et al. (2019) Offline impact of transcranial focused ultrasound on cortical activation in primates. Elife 8:e40541. 
bioRxiv preprint doi: https://doi.org/10.1101/2021.10.09.463785; this version posted October 9, 2021. The copyright holder for this preprint (which was not certified by peer review) is the author/funder. All rights reserved. No reuse allowed without permission.

Wang H, Zhou X, Cui D, Liu R, Tan R, Wang X, Liu Z, Yin T (2019) Comparative study of transcranial magneto-acoustic stimulation and transcranial ultrasound stimulation of motor cortex. Frontiers in behavioral neuroscience 13:241.

Ward AR (2009) Electrical stimulation using kilohertz-frequency alternating current. Physical therapy 89:181-190.

Zesiewicz TA, Chari A, Jahan I, Miller AM, Sullivan KL (2010) Overview of essential tremor. Neuropsychiatric disease and treatment 6:401. 